\title{
Características de adsorção de fósforo em alguns solos da Amazônia Central.
}

\author{
Newton Paulo de Souza FALCÃO ${ }^{1}$, José Risonei Assis da SILVA ${ }^{1}$
}

\begin{abstract}
RESUMO
Com o objetivo de determinar as características de adsorção de fósforo utilizando-se a isoterma de Langmuir, e suas relações com algumas propriedades físicas e químicas de solos, foi desenvolvido um estudo no Laboratório de Solos do Instituto Nacional de Pesquisas da Amazônia (INPA), com amostras da camada superficial $(0-20 \mathrm{~cm})$ de oito solos do Estado do Amazonas. Grande variação na capacidade máxima de adsorção (CMAP), energia de adsorção e fator capacidade de P máximo $\left(\mathrm{FCP}_{\text {ín }}\right.$ ) foi observado. O Plintossolo Argilúvico alumínico (FTa) foi o solo que apresentou os maiores valores de CMAP e FCP maxx $_{\text {ax }}$ e o Latossolo Amarelo1 (LAx -1) apresentou o maior valor de energia de adsorção. A CMAP variou de 0,297 a $0,888 \mathrm{mg} \mathrm{P} \mathrm{g}^{-1}$ de solo, a energia de adsorção ficou na faixa de 0,230 a $0,730 \mathrm{~L} \mathrm{mg}^{-1}$, e o FCP máx $_{\text {variou de }}$ $137 \mathrm{a}^{2} 593 \mathrm{~mL} \mathrm{~g}^{-1}$. O parâmetro $\mathrm{FCP}_{\text {max }}$ obedeceu à seguinte ordem decrescente: Plintossolo Argilúvico alumínico $>$ Latossolo Amarelo $1>$ Latossolo Amarelo $3>$ Latossolo Amarelo $6>$ Latossolo Amarelo $2>$ Latossolo Amarelo $4>$ Argissolo Amarelo distrófico > Latossolo Amarelo 5. A CMAP e o $\mathrm{FCP}_{\text {max }}$ correlacionaram-se positivamente com o conteúdo de argila $\left(\mathrm{r}=0,948^{* *}\right.$ e $\left.\mathrm{r}=0,962^{* *}\right)$ e alumínio trocável $\left(\mathrm{r}=0,688^{*}\right.$ e $\left.\mathrm{r}=0,666^{*}\right)$, e negativamente com a saturação de bases $(\mathrm{r}$ $=-0,667^{*}$ e $\left.r=-0,761 *\right)$, respectivamente. A energia de adsorção apresentou correlação positiva com o conteúdo de $\operatorname{argila}\left(r=0,783^{*}\right)$ e negativa com a saturação de bases $\left(r=-0,775^{*}\right)$.
\end{abstract}

\section{PALAVRAS-CHAVE}

Fixação de P, Solos tropicais, Amazônia

\section{Phosphorus adsorption characteristics in some Central Amazonian soils.}

\begin{abstract}
The objective of this paper was to determine the phosphorus adsorption characteristics using the Langmuir isotherm and it is relation with some physical and chemical properties of soils. The study was carried out in the Soil and Plant Laboratory, of the National Institute for Amazon Research (INPA), Brazil, with surface $(0-20 \mathrm{~cm})$ samples from eight soils of the Amazonas State. Ample variation of the maximum phosphorus capacity factor (MPAC), adsorption energy and maximum phosphorus capacity factor (PMCF) were observed. The Plinthite soil showed the highest values of the MPAC and PMCF and the Yellow Oxisol 1 (LAx-1) showed the highest adsorption energy. The MPAC ranged from 0.297 to $0.888 \mathrm{mg} \mathrm{P}^{-1}$ of soil, the adsorption energy with which the phosphorus is sorbed was between 0,230 and $0,730 \mathrm{~L} \mathrm{mg}^{-1}$, and the PmCF ranged from 137 to $593 \mathrm{~mL} \mathrm{~g}$. The PmCF decreased from Plinthite (FTa) > Yellow Oxisol (LAx-1) > Yellow Oxisol (LAx-3) > Yellow Oxisol (LAx-6) > Yellow Oxisol (LAx-2) > Yellow Oxisol (LAx-4) > Yellow Ultisol (PAd) $>$ Yellow Oxisol (LAx-5). The MPAC and the PmCF were positively correlated with clay content $\left(r=0,948^{*}\right.$ e $r=$ $0,962 *)$ and exchangeable $\mathrm{Al}^{\mathrm{B}}\left(r=0,688^{*}\right.$ e $\left.r=0,666^{*}\right)$, and negatively correlated with base saturation $\left(r=-0,667^{*}\right.$ er $r=-0,761 *)$, respectively. The adsorption energy was positively correlated with clay content $\left(r=0,783^{*}\right)$ and negatively with base saturation $(r=-0,775 \%)$.
\end{abstract}

KEY WORDS

Phosphorus fixation, tropical soil. Amazonia

${ }^{1}$ Instituto Nacional de Pesquisas da Amazônia, INPA/CPCA, Cx. Postal 478, 69011-970 Manaus, AM, Brasil. e-mail: nfalcao@inpa.gov.br, risonei@inpa.gov.br 


\section{ACTA \\ AMAZONICA}

CARACTERÍSTICAS DE ADSORÇÃO DE FÓSFORO

EM ALGUNS SOLOS DA AMAZỐNIA CENTRAL

\section{INTRODUÇÃO}

As principais limitações de fertilidade dos solos da Amazônia são a acidez elevada, baixa capacidade de troca de cátions, deficiência de $\mathrm{N}, \mathrm{P}$ K, S, Ca, Mg, B, Cu, Zn e também a alta capacidade desses solos para fixar o $\mathrm{P}$ aplicado como fertilizante (Sanchez \& Cochrane, 1980). O teor total de $\mathrm{P}$ dos solos se situa, de modo geral, entre 200 e $3000 \mathrm{mg}$ $\mathrm{kg}^{-1}$ de $\mathrm{P}$, sendo que menos de $0,1 \%$ desse total encontra-se na solução do solo. Em solos agrícolas, os valores de P em solução estão, com freqüência, entre 0,002 e $2 \mathrm{mg} \mathrm{L}^{-1}$ de P. Há diferenças entre solos, quanto à eficiência de utilização de fósforo pelas plantas, relacionadas com processos de adsorção (Fardeau, 1996).

Estima-se que apenas $5 \%$ a $25 \%$ do fósforo solúvel adicionado ao solo, como adubo, seja aproveitado pela cultura que o recebeu e que $95 \%$ a $75 \%$ dele seja fixado. $\mathrm{O}$ termo fixação de P envolve mecanismos de adsorção, através de ligações eletrostáticas ou covalentes, e de precipitação, com formação de compostos insolúveis, tornando o elemento indisponível para as plantas (Alcarde et al., 1991). A representação matemática dos mecanismos de adsorção e precipitação tem sido realizada através de isotermas de adsorção, que descrevem quantitativamente a adsorção de solutos à superfície de sólidos, sob condições constantes de temperatura e pressão, mostrando a quantidade de adsorvato sorvido em função de uma concentração de equilíbrio (Bohn et al., 1985). Existem, segundo Barrow (1978), duas boas razóes para utilizar modelos que descrevem a adsorção no solo. A primeira é que possibilita a expressão numérica das propriedades de solo e, a segunda, que permite conhecer mais a respeito da natureza dos processos de adsorção.

A isoterma de Langmuir foi utilizada pela primeira vez por Olsen \& Watanabe (1957) para descrever a adsorção de $P$ pelo solo. A maior vantagem da equação de Langmuir, sobre outras isotermas, é que a capacidade máxima de adsorção de $\mathrm{P}$ (CMAP) e a constante relacionada com a energia de adsorção podem ser calculadas e estes valores podem ser relacionados com várias propriedades do solo, que fornecerão informações sobre a natureza da reação entre o solo e o fertilizante fosfatado (Olsen \& Watanabe, 1957; Novais \& Smyth, 1999).

A CMAP revela o que poderá ocorrer com o $\mathrm{P}$ adicionado ao solo para as culturas, em termos de aproveitamento e, também, para utilização posterior pelas plantas, constituindose no que se denomina de "efeito residual" (Pereira \& Faria, 1998). Segundo Woodruff \& Kamprath (1965), o valor da CMAP de solos tem sido usado como uma estimativa da quantidade desse nutriente requerida pelos solos para condicionar o crescimento máximo das plantas.

Segundo Novais \& Smyth (1999), o fator capacidade do P (FCP) pode ser entendido como a resistência do solo a mudanças no fator intensidade (I) quando se põe ou se retira P do solo $(\mathrm{Q})$. O FCP $(\mathrm{Q} / \mathrm{I})$ é definido pelo equilíbrio ou "ligação" entre variação de quantidade e variação de intensidade. Diversas medidas, ou índices, do FCP, definido pela relação $\mathrm{Q} / \mathrm{I}$, são obtidas a partir das isotermas de adsorção, uma vez ser o FCP controlado pelos processos de adsorção/dessorção do P do solo (Holford \& Mattingly, 1976; Holford, 1979).

Constantes da isoterma de Langmuir - CMAP e "energia de adsorção" - correlacionam-se com o FCP, ou índices com ele relacionados (Novais, 1977; Muniz et al.,1987; Novais et al., 1993). Holford \& Mattingly (1976) propuseram a Capacidade Tampão Máxima, enquanto que Novais \& Smyth (1999) propuseram o Fator Capacidade de P Máximo $\left(\mathrm{FCP}_{\text {máx }}\right)$. De acordo com Novais \& Smyth (1999), o FCP ${ }_{\text {máx. }}$ integra uma medida de quantidade, capacidade máxima de adsorção de P (CMAP) e uma de qualidade ("energia de adsorção") ou, segundo Holford \& Mattingly (1976), integra uma medida extensiva e uma intensiva, respectivamente.

Este trabalho teve como objetivos estudar três parâmetros de adsorção de fósforo (capacidade máxima de adsorção de fósforo, constante de energia de adsorção e fator capacidade de $\mathrm{P}$ máximo ou capacidade tampão máxima de P), com a equação de Langmuir, em solos característicos do Estado do Amazonas, correlacionandoos com algumas propriedades físicas e químicas apresentadas pelos referidos solos.

\section{MATERIAL E MÉTODOS}

O estudo foi desenvolvido durante o período de Agosto de 2000 a julho de 2001 no Laboratório Temático de Solos e Plantas do Instituto Nacional de Pesquisas da Amazônia. Para isto, foram coletadas amostras da camada superficial $(0-20 \mathrm{~cm})$ de oito classes de solos do Estado do Amazonas: dois Latossolos Amarelos distrofico (LAx-1 e LAx-2) e um Argissolo Amarelo distrófico (PAd), coletados no município de Manacapuru; quatro Latossolos Amarelos coesos distróficos (LAx-3, LAx-4, LAx-5 e LAx-6) provenientes de Borba e uma Laterita Hidromórfica ou Plintossolo Argilúvico alumínico (FTa) de Humaitá, cujas características químicas e físicas, constam na Tabela 1 , segundo método de análise da EMBRAPA (1999).

Para a determinação dos valores da capacidade máxima de adsorção de P (CMAP), constante de energia de adsorção e o fator capacidade de $\mathrm{P}$ máximo $\left(\mathrm{FCP}_{\text {máx }}\right)$, foi utilizada a seguinte metodologia: amostras de $2,5 \mathrm{~g}$ de solo seco, passado em peneira de $2 \mathrm{~mm}$, foram agitadas por 24 horas, à temperatura ambiente, em $25 \mathrm{~mL}$ de solução de $\mathrm{CaCl}_{2}$ 0,01 M, contendo: 0, 20, 40, 60, 80 e $100 \mathrm{mg} \mathrm{L}^{-1}$ de $\mathrm{P}$ na forma de $\mathrm{KH}_{2} \mathrm{PO}_{4}$. Após o período de agitação, a suspensão foi centrifugada a $4500 \mathrm{rpm}$ e filtrada. Em seguida, alíquotas do extrato totalmente límpido foram usadas para determinação do fósforo pelo método baseado no emprego do complexo fosfomolíbdico usando o ácido ascórbico como redutor (Braga \& Defelipo, 1974). O fósforo adsorvido foi calculado pela diferença entre as quantidades de fósforo adicionadas e as quantidades encontradas na solução de equilíbrio (sobrenadante), após o período de agitação 


\section{ACTA AMAZONICA}

Tabela 1. Características químicas e físicas das amostras dos solos estudados (profundidade de 0-20 cm).

\begin{tabular}{|c|c|c|c|c|c|c|c|c|}
\hline \multirow{2}{*}{ Características } & \multirow{2}{*}{ PAd } & \multicolumn{7}{|c|}{ Solos } \\
\hline & & LAx-1 & LAx-2 & LAx-3 & LAx-4 & LAx-5 & LAx-6 & $\mathrm{FTa}$ \\
\hline $\mathrm{PH}$ em $\mathrm{H}_{2} \mathrm{O}(1: 2,5)$ & 4,45 & 4,31 & 4,38 & 4,02 & 4,33 & 4,11 & 4,04 & 4,71 \\
\hline $\mathrm{Ca}^{2}+\left(\mathrm{cmol}_{\mathrm{c}} \mathrm{kg}^{-1}\right)$ & 0,05 & 0,07 & 0,03 & 0,10 & 0,28 & 0,64 & 0,12 & 0,03 \\
\hline $\mathrm{Mg}^{2}+\left(\mathrm{cmol}_{\mathrm{c}} \mathrm{kg}^{-1}\right)$ & 0,04 & 0,03 & 0,04 & 0,08 & 0,08 & 0,26 & 0,09 & 0,04 \\
\hline $\mathrm{K}+\left(\mathrm{cmol}_{\mathrm{c}} \mathrm{kg}^{-1}\right)$ & 0,14 & 0,03 & 0,05 & 0,08 & 0,05 & 0,14 & 0,08 & 0,06 \\
\hline$P\left(\mathrm{mg} \mathrm{kg}^{-1}\right)$ & 4,18 & 9,17 & 2,53 & 9,37 & 9,25 & 16,38 & 7,92 & 0,68 \\
\hline $\mathrm{Al}^{3}+\left(\mathrm{cmol}_{\mathrm{c}} \mathrm{kg}^{-1}\right)$ & 0,70 & 1,05 & 1,10 & 1,70 & 0,80 & 1,10 & 1,75 & 4,28 \\
\hline $\mathrm{H}+\mathrm{Al}\left(\mathrm{cmol}_{c} \mathrm{~kg}^{-1}\right)$ & 4,90 & 7,85 & 7,65 & 8,45 & 5,95 & 9,50 & 8,20 & 7,06 \\
\hline $\mathrm{S}\left(\mathrm{cmol}_{\mathrm{c}} \mathrm{kg}^{-1}\right)^{*}$ & 0,23 & 0,13 & 0,12 & 0,26 & 0,41 & 1,04 & 0,29 & 0,13 \\
\hline $\mathrm{t}\left(\mathrm{cmol}_{\mathrm{c}} \mathrm{kg}^{-1}\right)^{* *}$ & 0,93 & 1,18 & 1,22 & 1,96 & 1,21 & 2,14 & 2,04 & 4,41 \\
\hline $\mathrm{T}\left(\mathrm{cmol}_{\mathrm{c}} \mathrm{kg}^{-1}\right)^{* * *}$ & 5,13 & 7,98 & 7,77 & 8,71 & 6,36 & 10,54 & 8,49 & 7,19 \\
\hline $\mathrm{V}(\%)^{* * * *}$ & 4,48 & 1,63 & 1,55 & 2,99 & 6,45 & 9,87 & 3,42 & 1,81 \\
\hline$C\left(\mathrm{~g} \mathrm{~kg}^{-1}\right)$ & 27,0 & 19,0 & 30,0 & 45,3 & 39,9 & 18,5 & 38,2 & 17,50 \\
\hline Argila $\left(\mathrm{g} \mathrm{kg}^{-1}\right)$ & 134 & 495 & 366 & 267 & 213 & 177 & 324 & 522 \\
\hline Silte $\left(\mathrm{g} \mathrm{kg}^{-1}\right)$ & 58 & 52 & 30 & 121 & 155 & 139 & 98 & 189 \\
\hline Areia $\left(\mathrm{g} \mathrm{kg}^{-1}\right)$ & 808 & 453 & 604 & 612 & 632 & 684 & 578 & 289 \\
\hline
\end{tabular}

* Soma de bases trocáveis; ${ }^{* *}$ Capacidade de troca de cátions efetiva; ${ }^{* * *}$ Capacidade de troca de cátions potencial; ${ }^{* * *}$ Saturação de bases.

A partir dos dados obtidos, construiu-se isotermas de adsorção, plotando-se a quantidade de fósforo adsorvido, na ordenada, e a concentração na solução de equilíbrio na abcissa. A forma hiperbólica da equação de Langmuir é dada pela seguinte expressão:

$$
\mathrm{x} / \mathrm{m}=(\mathrm{abC}) /(1+\mathrm{aC})
$$

em que $\mathrm{x} / \mathrm{m}$ é a quantidade de $\mathrm{P}$ adsorvido ao solo, em $\mathrm{mg} \mathrm{P}$ (x)/g solo (m); b é a CMAP do solo, em $\mathrm{mg} \mathrm{g}^{-1}$ de P no solo; C é a concentração de $\mathrm{P}$ na solução de equilíbrio (sobrenadante), em $\mathrm{m} \mathrm{L} \mathrm{L}^{-1}$, e a é a constante relacionada com a energia de adsorção do elemento ao solo, em $\left(\mathrm{mg} \mathrm{L}^{-1}\right)^{-1} \mathrm{ou}$ $\mathrm{L} \mathrm{mg}^{-1}$ (Olsen \& Watanabe, 1957).

Para estimativas das constantes a e b, a equação hiperbólica de Langmuir foi transformada em sua forma linear, que corresponde $\mathrm{a}$ :

$$
\mathrm{C} /(\mathrm{x} / \mathrm{m})=1 /(\mathrm{ab})+(1 / \mathrm{b}) \mathrm{C}
$$

A capacidade máxima de adsorção do fósforo (b) foi determinada pelo valor inverso da declividade da reta, a constante de energia de adsorção (a) pela relação entre a declividade da reta e a interseção da mesma com o eixo das ordenadas e o fator capacidade de $\mathrm{P}$ máximo $\left(\mathrm{FCP}_{\text {maxx }}\right.$ ) pelo produto entre a capacidade máxima de adsorção de $\mathrm{P}$ e a energia de adsorção segundo Bahia Filho et al. (1983a) e Novais \& Smyth (1999).

\section{RESULTADOS E DISCUSSÃO}

Os resultados das análises físicas e químicas dos solos, contidos na Tabela 1, indicam que os valores de $\mathrm{pH}$ dos solos avaliados apresentam-se extremamente baixos, característicos dos solos ácidos. A soma de bases trocáveis dos solos ficou abaixo de $1 \mathrm{cmol}_{\mathrm{c}} \mathrm{kg}^{-1}$, com exceção do LAx5, que apresentou $1,04 \mathrm{cmol}_{\mathrm{c}} \mathrm{kg}^{-1}$. Os teores de carbono estão na faixa de $17,50 \mathrm{~g} \mathrm{~kg}^{-1}$ (FTa) a $45,3 \mathrm{~g} \mathrm{~kg}^{-1}$ (LAx-3). O P disponível variou de $0,68 \mathrm{mg} \mathrm{kg}^{-1}$ no FTa a $16,38 \mathrm{mg} \mathrm{kg}^{-1}$ no LAx-5. Os teores de Alumínio trocável estão na faixa de 0,70 $\mathrm{cmol}_{\mathrm{c}} \mathrm{kg}^{-1}$ (PAd) a $4,28 \mathrm{cmol}_{\mathrm{c}} \mathrm{kg}^{-1.3}$ (FTa) contribuindo com mais de $97 \%$ para elevação da CTC efetiva deste solo que alcançou $4,41 \mathrm{cmol}_{\mathrm{c}} \mathrm{kg}^{-1} \mathrm{dm}^{-3}$. Portanto, os solos estudados apresentam extrema pobreza de nutrientes $(\mathrm{Ca}, \mathrm{Mg}, \mathrm{K})$ e acidez excessiva. Os valores da CTC efetiva (muito baixo) refletem que estes solos, sob condições naturais ácidas, apresentam baixa capacidade de reter cátions. Quanto à textura observou-se que vai de arenosa (PAd), a média (LAx3, LAx-4, LAx-5, LAx-6) e argilosa (LAx-1, LAx-2, FTa).

As isotermas de adsorção de fósforo (Figuras 1 e 2) correspondentes a oito solos estudados, demonstraram que as quantidades de fosfatos adsorvidas pelos solos aumentaram com a concentração da solução de equilíbrio, inicialmente de uma maneira mais intens. Porém, tenderam a adquirir um valor constante a altas concentrações. As curvas de adsorção não apresentadas nas Figuras 1 e 2 são semelhantes ou intermediárias às curvas de adsorção plotadas. As formas das isotermas são características da adsorção química de uma monocamada aniônica, pela fase sólida adsorvente do solo, concordando com Barrow (1961). Na fase inicial de rápida adsorção de P no solo, há uma atração eletrostática inicial (Barrow, 1985), seguida pela adsorção por oxidróxidos, por meio de troca de ligantes (Parfitt, 1978; Barrow, 1985; Sanyal \& De Datta, 1991). Segundo Novais e Smyth (1999), nessa reação, denominada quimiossorção, há troca de ligantes, 


\section{ACTA \\ AMAZONICA}

CARACTERÍSTICAS DE ADSORÇÃO DE FÓSFORO

EM ALGUNS SOLOS DA AMAZỐNIA CENTRAL como $\mathrm{OH}^{-}$e $\mathrm{OH}_{2}^{+}$, da superfície dos óxidos, por fosfato da solução. É uma ligação predominantemente covalente ("adsorção específica"), ao contrário do $\mathrm{NO}^{3-}$ ou do $\mathrm{Cl}$, adsorvidos por atração eletrostática ("adsorção não específica”) (Parfitt et al., 1975; Parfitt, 1978).

Houve uma ampla variação dos valores de CMAP, energia de adsorção e $\mathrm{FCP}_{\text {maxx }}$, em função do solo estudado (Tabela 2). Constatou-se que o Plintossolo Argilúvico alumínico (FTa) apresentou o maior valor de CMAP. Com valores intermediários de CMAP encontraram-se os Latossolos Amarelos coesos (LAx), enquanto que o valor mais baixo foi observado para o Argissolo Amarelo distrófico (PAd). Essa variação pode estar diretamente ligada à composição do solo e indiretamente à influência de alguns dos componentes na retenção do fósforo. Em vista disto, os valores da CMAP, energia de adsorção e $\mathrm{FCP}_{\text {máx. }}$ foram correlacionados com algumas características dos solos (Tabela 3), para a obtenção de informações a respeito de quais constituintes podem estar atuando no processo de retenção do fósforo.

A CMAP apresentou correlação positiva com o teor de argila $\left(r=0,95^{* *}\right)$, o alumínio trocável $(r=0,69 *)$, e negativa com a saturação de bases $\left(r=-0,66^{*}\right)$. Tucci (1991) verificou que aumentando os teores de argila ocorre o aumento da CMAP em solos de Manaus. $\mathrm{O}$ autor menciona que variações na capacidade de retenção de fósforo de diferentes solos, com teores de argila semelhante, são, provavelmente, devido a variações na composição mineralógica da fração argila dos solos. Sá Jr. et al. (1968), avaliando a retenção de fósforo em 17 solos de Pernambuco, também verificaram que os valores da CMAP foram maiores para os solos com textura argilosa, enquanto que os com textura arenosa apresentaram valores menores.

$\mathrm{O}$ aumento da CMAP à medida que aumentam os teores de alumínio trocável do solo foi também verificado por Coleman et al. (1960), que observaram alta correlação $(\mathrm{r}=0,838)$ entre a CMAP e o Al ${ }^{3+}$ em solos da Carolina do Norte (EUA). Esse resultado concorda também com as observações de Syers et al. (1971) em

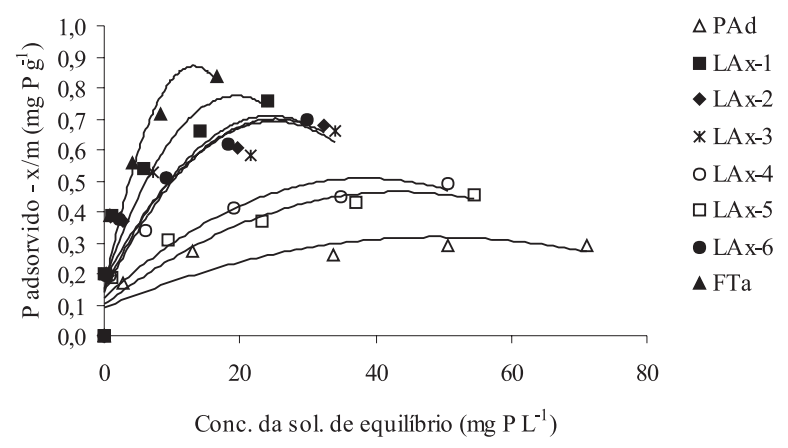

Figura 1 - Isotermas de adsorção de fósforo de oito quatro solos do Estado do Amazonas.
Ultissolos e Oxissolos do Rio Grande do Sul $(r=0,840)$, mas diverge dos dados de Leal \& Velloso (1973), que não observaram correlação entre Al trocável e fixação de fósforo em nove solos do cerrado.

A correlação negativa entre a CMAP e a saturação de bases verificado neste trabalho diverge dos resultados obtidos por Pereira \& Faria (1998), que observaram um aumento da adsorção do $\mathrm{P}$ à medida que aumentava $\mathrm{a}$ saturação de bases. Woodruff \& Kamprath (1965), relacionando com a disponibilidade de $\mathrm{P}$ para as plantas, observaram que a adição de calcário em solos que tinham alta saturação de Al trocável reduziu significativamente a CMAP quando calculado através da isoterma de Langmuir.

Baixas correlações foram encontradas entre a CMAP e o teor de carbono orgânico, pH e H + Al. Na Tabela 3, percebe-se ainda, que a energia de adsorção apresentou correlação positiva com o conteúdo de argila $(\mathrm{r}=$ $\left.0,783^{*}\right)$. Este resultado concorda com os obtidos por Novais \& Kamprath (1979).

O valor da CMAP tem sido utilizado no processo de recomendação de adubação fosfatada (Woodruff \& Kamprath, 1965). Para solos do cerrado, a produção máxima tem sido obtida quando a quantidade de $\mathrm{P}$ aplicado varia entre 0,4 e 1,3 vez o valor da CMAP do solo (Braga \& Defelipo, 1972; Bahia Filho \& Braga, 1975b; Vasconcelos et al., 1975). No entanto, segundo Novais \& Kamprath (1979b), o uso apenas da CMAP para predizer o requerimento de $\mathrm{P}$ dos solos para $\mathrm{O}$ crescimento de plantas não parece ser adequado, uma vez que ambos os fatores, intensidade e capacidade, pelo menos, são requeridos nos processos de predição das respostas das plantas à adubação fosfatada. De acordo com Novais \& Smyth (1999), a utilização de apenas o valor da CMAP, com sucesso, em solos de cerrado parece estar relacionada aos valores iniciais semelhantes de intensidade (muito baixos, praticamente zero $\mu \mathrm{g} \mathrm{g}^{-1}$ de P na solução do solo). Neste caso, a adubação fosfatada requerida torna-se função de apenas da CMAP como medida do fator capacidade.

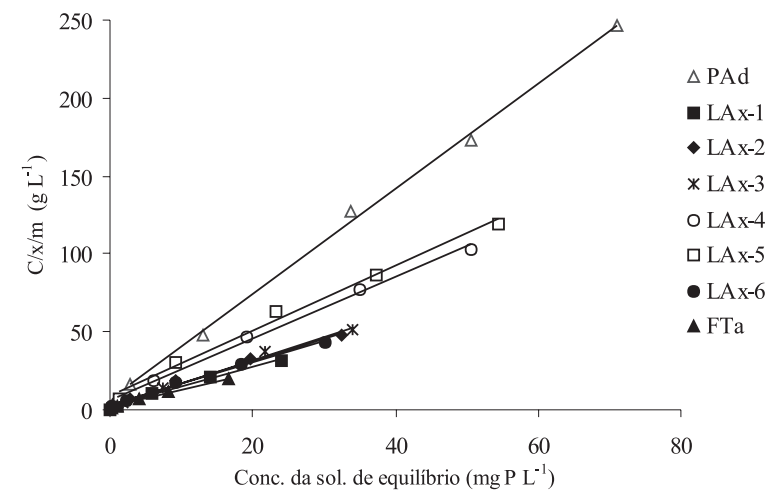

Figura 2 - Isotermas de adsorção de Langmuir na forma linear dos oito solos estudados. 


\section{ACTA AMAZONICA}

Tabela 2 - Equações da isoterma de Langmuir, capacidade máxima de adsorção de P (CMAP), energia de adsorção e fator capacidade de P máximo $\left(\mathrm{FCP}_{\text {máx }}\right)$ para os diversos solos estudados

\begin{tabular}{|c|c|c|c|c|c|c|}
\hline Solos & Procedência & $\begin{array}{c}\text { Equação de regressãoC } /(\mathrm{x} / \mathrm{m})=\mathrm{A} \\
+\mathrm{BX} ;(\mathrm{X}=\mathrm{P} \text {-solução })\end{array}$ & $\mathrm{R} 2$ & CMAP mg g ${ }^{-1}$ & $\begin{array}{l}\text { Energia de } \\
\text { adsorção L mg-1 }\end{array}$ & FCPmáx.mL g-1 \\
\hline PAd & Manacapuru & $Y=7,2696+3,3605 X$ & $0,997^{* *}$ & 0,2975 & 0,4623 & 137 \\
\hline LAx-1 & Manacapuru & $Y=1,7639+1,2879 X$ & $0,988^{* *}$ & 0,7764 & 0,7301 & 567 \\
\hline LAx-2 & Manacapuru & $Y=3,4396+1,4125 X$ & $0,993^{* *}$ & 0,7079 & 0,4106 & 291 \\
\hline LAx-3 & Borba & $Y=2,1561+1,5008 X$ & $0,994^{* *}$ & 0,6663 & 0,6961 & 464 \\
\hline LAx-4 & Borba & $Y=5,8860+1,9726 X$ & $0,995^{* *}$ & 0,5069 & 0,3351 & 170 \\
\hline LAx-5 & Borba & $Y=9,0521+2,0848 X$ & $0,991^{* *}$ & 0,4797 & 0,2303 & 111 \\
\hline LAx-6 & Borba & $Y=3,1424+1,3740 X$ & $0,988^{* *}$ & 0,7278 & 0,4373 & 318 \\
\hline $\mathrm{FTa}$ & Humaitá & $Y=1,6871+1,1261 X$ & $0,989 * *$ & 0,8880 & 0,6675 & 593 \\
\hline
\end{tabular}

${ }^{* *}$ significativo a $1 \%$

Tabela 3 - Coeficientes de correlação linear simples ( r ) entre os parâmetros de adsorção e algumas características dos solos estudados

\begin{tabular}{cccc}
\hline \hline Características dos solos & CMAP & $\begin{array}{c}\text { Energia de } \\
\text { adsorção }\end{array}$ & FCPmáx \\
\hline Argila & $0,948^{* *}$ & $0,783^{*}$ & $0,962^{* *}$ \\
\hline Carbono Orgânico & $-0,137 \mathrm{~ns}$ & $-0,042 \mathrm{~ns}$ & $-0,167 \mathrm{~ns}$ \\
pH em H2O (1:2,5) & $0,170 \mathrm{~ns}$ & $0,221 \mathrm{~ns}$ & $0,259 \mathrm{~ns}$ \\
Saturação de bases (V) & $-0,667^{*}$ & $-0,775^{*}$ & $-0,761^{*}$ \\
Alumínio trocável (Al3+ & $0,688^{*}$ & $0,456 \mathrm{~ns}$ & $0,666^{*}$ \\
\hline $\mathrm{H}+$ Al & $0,416 \mathrm{~ns}$ & $0,028 \mathrm{~ns}$ & $0,200 \mathrm{~ns}$ \\
\hline \hline
\end{tabular}

"* Significativo a 1\%; " Significativo a 5\%; (ns) Não significativo.

Examinando os resultados da Tabela 3, verifica-se que o $\mathrm{FCP}_{\text {máx }}$ apresentou correlação positiva com o conteúdo de $\operatorname{argila}(r=0,962 * *)$ e alumínio trocável $\left(r=0,666^{*}\right)$ e correlação negativa com a saturação de bases $(r=-0,761 *)$ . $\mathrm{OFCP}_{\text {máx. }}$ obedeceu à seguinte ordem decrescente: $\mathrm{FTa}>$ LAx-1 > LAx-3 > LAx-6 > LAx-2 > LAx-4 > PAd > LAx-5. Estes resultados concordam, de maneira geral, com a escala (hipotética) decrescente de importância do fator capacidade de P (FCP) proposta por Novais \& Smyth (1999): FCP de solos argilosos pobres em $\mathrm{P}>\mathrm{FCP}$ de solos argilosos ricos em P > FCP de solos arenosos pobres em P > FCP de solos arenosos ricos em $P$.

Segundo Novais \& Smyth (1999), talvez fique mais clara a razão de ter uma medida extensiva (quantidade adsorvida) e outra intensiva (qualidade de adsorção), como propostas por Holford \& Mattingly (1976), denominada $\mathrm{FCP}_{\text {máx }}$ (CMAP $\mathrm{x}$ "energia de adsorção"). Como a CMAP tende a ser constante, e a "energia de adsorção" varia com o "status" de $\mathrm{P}$ do solo, pode-se imaginar ter, na "energia de adsorção"ou em alguma medida de dessorção de $\mathrm{P}$ do solo, uma maneira de comparar comportamentos de P num mesmo solo, com diferentes históricos de uso. Portanto, medidas extensivas, como a capacidade máxima de adsorção de $\mathrm{P}$, deverão continuar sendo importantes na comparação entre solos como critério de previsão do comportamento do $\mathrm{P}$, desde que esses solos tenham suas medidas intensivas em seus valores máximos (solos pobres em $\mathrm{P}$, como a maioria dos solos Amazônicos, e "energia de adsorção” em valores próximos do máximo).

\section{CONCLUSÕES}

Houve grande variação da capacidade máxima de adsorção (CMAP), energia de ligação e fator capacidade de P ( FCP $_{\text {máx }}$ ) máximo pelos solos estudados, sendo que o Plintossolo Argilúvico alumínico foi que apresentou os

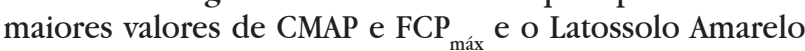
coeso 1 apresentou o maior valor de energia de adsorção.

A CMAP variou de 0,297 a $0,888 \mathrm{mg} \mathrm{P} \mathrm{g}^{-1}$ de solo, a energia com que o fósforo é retido ficou na faixa de 0,230 a $0,730 \mathrm{~L}$ $\mathrm{mg}^{-1}$ e o $\mathrm{FCP}_{\text {máx }}$ variou de 137 a $593 \mathrm{~mL} \mathrm{~g}^{-1}$. O parâmetro $\mathrm{FCP}_{\text {máx }}$ obedeceu à seguinte ordem decrescente: FTa $>$ LAx-1 $>$ LAX-3 > LAx-6 > LAx-2 > LAx-4 > PAd > LAx-5.

A CMAP e o $\mathrm{FCP}_{\text {máx }}$ correlacionaram-se positivamente com o conteúdo de argila e alumínio trocável e, negativamente com a saturação de bases. A energia de ligação apresentou correlação positiva com o conteúdo de argila e negativa com a saturação de bases.

\section{BIBLIOGRAFIA CITADA}

Alcarde, J.C.; Guidolin, J.A., Lopes, A.S. 1991. Os Adubos e a eficiência das adubações. 2. ed. ANDA, São Paulo. 35p.

Bahia Filho, A.F.C.; Braga, J.M. 1975b. Fósforo em Latossolos do Estado de Minas Gerais. III. Índices de disponibilidade de fósforo e crescimento vegetal. Experimentiae, 20:217-234. 


\section{ACTA AMAZONICA}

CARACTERÍSTICAS DE ADSORÇÃO DE FÓSFORO EM ALGUNS SOLOS DA AMAZỐNIA CENTRAL
Bahia Filho, A.F.C.; Braga, J.M.; Ribeiro, A.C.; Novais, R.F. 1983a. Sensibilidade de extratores químicos à capacidade tampão de fósforo. R. Bras. Ci. Solo, 7:243-249.

Barrow, G.M. 1961. Physical chemistry. McGraw-Hill, New York. 694p.

Barrow, N.J. 1978. The description of phosphorus adsorption curves. Soil Sci., 29:447- 462.

Barrow, N.J. 1985. Reaction of anions and cations with variablecharge soils. Adv. Agron., 38:183-230.

Bohn, H., Mcneal, B., O' Connor, G. 1985. Soil Chemistry. J. Wiley , Toronto. 341p.

Braga, J.M.; Defelipo, B.V. 1972. Relações entre formas de fósforo inorgânico, fósforo disponível e material vegetal em solos sob vegetação de cerrado: I. Trabalhos de laboratório. $R$. Ceres, 19:124-136.

Braga, J.M.; Defelipo, B.V. 1974. Determinação espectrofotométrica de fósforo em extratos de solo e material vegetal. $R$. Ceres, 21:73-85.

Coleman, N.T.; Thorup, J.T.; Jackson, W.A. 1960. Phosphate Sorption Reations That Involve Exchangeable Al. Soil Sci., 90:1-7.

EMBRAPA. 1999. Manual de análises químicas de solos, plantas e fertilizantes. Embrapa, Brasília. 370p.

Fardeau, J.C. 1996. Dynamics of phosphate in soils. An isotopic outlook. Fert. Res., 45:91-100.

Holford, I.C.R. 1979. Evaluation of soil phosphate buffering indices. Aust. J.Soil Res., 17:495-504.

Holford, I.C.R.; Mattingly; G.E.G. 1976. Phosphate adsorption and plant avaialability of phosphate. Plant Soil, 44:377-389.

Leal, J.R.; Velloso, A.C.X. 1973. Adsorção de fosfatos em Latossolos sob vegetação de cerrado. Pesq. agropec. bras., Sér. Agr., 8:81-88.

Muniz, A.S.; Novais, R.F.; Freire, F.M.; Neves, J.C.L.; Barros, N.F. 1987. Disponibilidade de fósforo e recomendação de adubação avaliadas por meio de extratores químicos e do crescimento de soja em amostras de solo com diferentes valores do fator capacidade. $R$. Ceres, 34:125-151.

Novais, R.F. 1977. Phosphorus supplying capacity of previously heavily fertilized soils. Tese de Doutorado, North Carolina State University, Releigh. 153p.

Novais, R.F.; Kamprath, E.J. 1979. Parâmetros das isotermas de adsorção de fósforo como critério de recomendação de adubarão fosfatada. $R$. bras. Ci. Solo, 3(1):37-41.

Novais, R.F.; Neves, J.C.L.; Barros, N.F.; Casali, V.W.D.; Fabres, A.S. 1993. The influence of the soil phosphate capacity factor on soil and plant phosphorus critical levels of different vegetables. In: Fragoso, M.A.C.; Beusichem, M.L.V. (Eds). Optimization of plant nutrition. Kluwer Academic Publishers, Netherlands. p.73-76.
Novais, R.F.; Smyth, T.J. 1999. Fósforo em solo e planta em condições tropicais. Universidade Federa de Viçosa, Viçosa, MG. 399p.

Olsen, E.R.; Watanabe, F. S. 1957. A method to determine a phosphorus adsorption maximum of soil as measured by the langmuir isotherm. Soil Sci. Soc. Am. proc., 21: 144-149.

Parfitt, R.L. 1978. Anion adsorption by soils and soil materials. Adv. Agron., 30:1-50.

Parfitt, R.L. Atkinson, R.J.; Smart, R.S.T.C. 1975. The mechanism of phosphate fixation by iron oxides. Soil Sci. Soc. Am. Proc., 39:837-841.

Pereira, J.R.; Faria, C.M.B. 1998. Sorção de fósforo em alguns solos do Semi-árido do Nordeste brasileiro. Pesq. agropec. bras., 33(7):1179-1184.

Sá Jr., J.P.N. Gomes, I.F., Vasconcelos, A.L. de. 1968. Retenção de fósforo em solos da Zona da Mata de Pernambuco. Pesq. agropec. bras., 3:183:188.

Sanchez, P.A.; Cochrane, T.T. 1980. Soils constraints in relation to major farming systems of tropical America. International Rice Research Institute, Los Banos. p.106-139.

Sanyal, S.K.; De Datta, S.K. 1991. Chemistry of phosphorus transformations in soil. Adv. Soil Sci., 16:1-120.

Syers, J.K.; Evans, T.D.; Williams, J.D.H.; Murdock, J.T. 1971. Phosphate sorption parameters of representative soils from Rio Grande do Sul, Brasil. Soil Sci., 112:267-275.

Tucci, C.A.F. 1991. Disponibilidade de fósforo em solos da Amazônia. Tese de doutorado, Universidade Federal de Viçosa. Viçosa, MG. 142p.

Vasconcelos, C.A.; Braga, J.M.; Novais, R.F.; Pinto, O.C.B. 1975. Fósforo em dois Latossolos do Estado de Mato Grosso: III. Relações entre planta, solo e fósforo. $R$. Ceres, 22:22-49.

Woodruff, J.R.; Kampratt, E.J. 1965. Phosphorus adsortion as maximum measured by the langmuir isotherm and its realtion hip to phosphorus availability, Soil. Sci. Soc. Am. Proc., 29:148150 .
RECEBIDO EM 23/10/2002
ACEITO EM 04/06/2004 